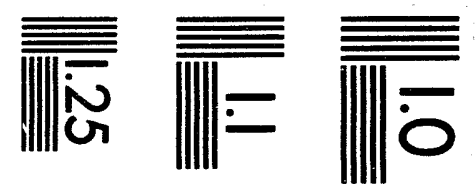

$$
\begin{aligned}
& \text { F) }
\end{aligned}
$$

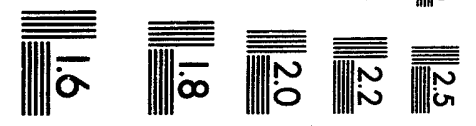



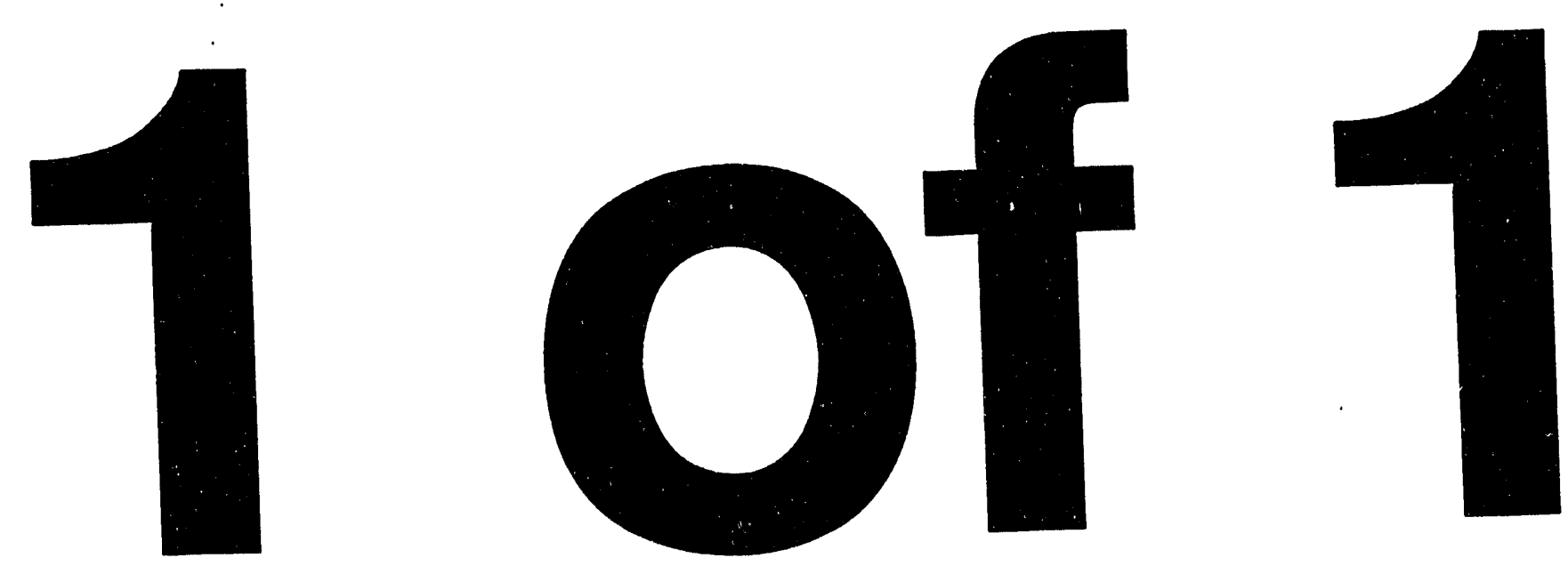


\section{Input Shaping for Three-Dimensional Slew Maneuvers of a Precision Pointing Flexible Spacecraft}

\author{
Clark R. Dohrmann ${ }^{1}$ \\ Sandia National Laboratories \\ Albuquerque, NM 87185-0439 \\ crdohrmesandia.gov
}

\author{
Rush D. Robinett \\ Sandia National Laboratories \\ Albuquerque, NM 87185-0314 \\ rdrobinesandia.gov
}

\begin{abstract}
A method is presented for input torque shaping for three-dimensional slew maneuvers of a precision pointing flexible spacecraft. The method determines the torque profiles for fixed-time, restto-rest maneuvers which minimize a specified performance index. Spacecraft dynamics are formulated in such a manner that the rigid body and flexible motions are decoupled. Furthermore, assembly of the equations of motion is simplified by making use of finite element analysis results. Input torque profiles are determined by solving an associated optimization problem using dynamic programming. Three example problems are provided to demonstrate the application of the method.
\end{abstract}

\section{Introduction}

The precision pointing of imaging satellites for nonproliferation has become an area of current interest. Imaging of ground targets for such applications leads to pointing accuracy requirements on the order of microradians. There are many contributors to pointing angle error, but vibrational disturbances caused by flexible, solar array support structures are potentially a major problem. In order to alleviate this problem, input shaping has been proposed to help minimize the residual vibration of the solar arrays after a threedimensional (3-D) slew maneuver.

It is only recently that input shaping for 3-D slew maneuvers of flexible spacecraft has appeared in

\footnotetext{
1 This work performed at Sandia National Laboratories supported by the U. S. Department of Energy under contract DE-AC04-94AL85000.
}

the technical literature [1]. The purpose of this paper is to present a method that applies to a wide range of spacecraft models. A key element of the approach is to express flexible motions in terms of the free-free mode shapes of the spacecraft. By doing so, the equations of motion can be easily assembled using the results of a finite element eigenanalysis.

Three example problems dealing with a simple spacecraft model are provided. The model consists of a rigid bus with two attached beams to model the effects of flexible solar panels. The effect of misalignment of the principal mass axes and the principal planes for bending is investigated. The results display interesting symmetries previously observed for planar maneuvers [2].

\section{Equations of Motion}

In this section, we develop equations of motion for flexible spacecraft subject to applied forces and moments. Flexible deformations are assumed to be small relative to the overall length of the spacecraft. The angular velocity magnitude for overall rigid body motion is also assumed to be small relative to the lowest structural natural frequency. Under these assumptions, the governing equations for rigid body and flexible motions can be decoupled. Furthermore, the equations can be assembled in a straightforward manner which makes use of finite element analysis results.

The spacecraft is idealized as a system of interconnected particles each of mass $m^{i}(i=$ $1, \ldots, N)$ as shown in Figure 1. Also shown in the figure are a floating reference frame, $B$, and an inertial frame, $A$. Orthogonal, dextral sets of unit vectors $\mathbf{b}_{1}, \mathbf{b}_{2}, \mathbf{b}_{3}$ and $\mathbf{a}_{1}, \mathbf{a}_{2}, \mathbf{a}_{3}$ are fixed in $B$ and 


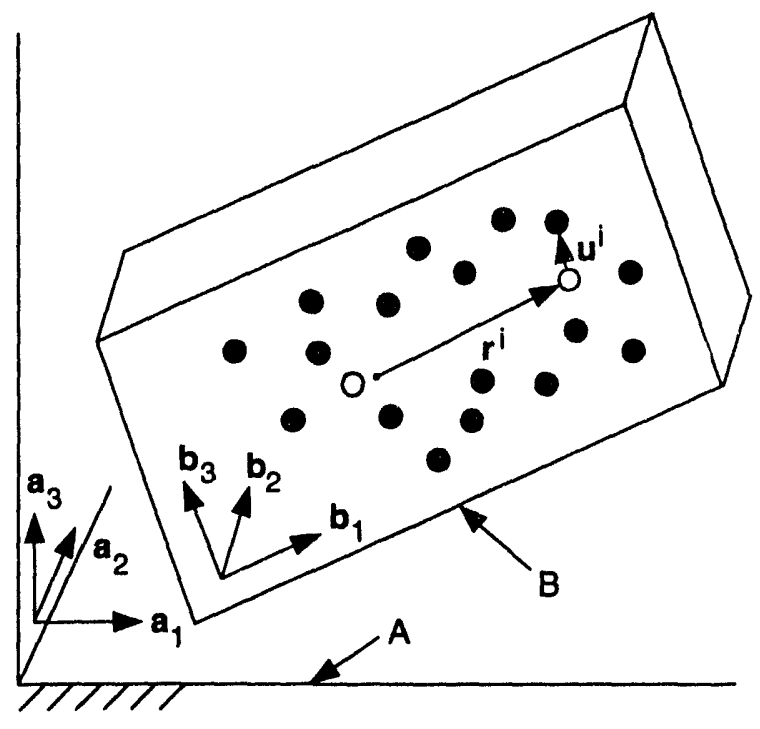

Figure 1: Sketch of system of particles and reference frames.

$A$, respectively. The angular velocity vector of $B$ in $A$ is denoted by $\boldsymbol{\omega}$. The position vector from the origin, $O$, of $B$ to the the $i$ 'th particle when the system is undeformed is denoted by $\mathbf{r}^{i}$. The displacement vector of the $i$ 'th particle from its undeformed position, $\mathbf{u}^{i}$, is assumed to be a function of generalized coordinates $q_{1}, \ldots, q_{n}$. The notational convention is adopted herein that for any vector, $\mathbf{v}$, one has $v_{k} \equiv \mathbf{v} \cdot \mathbf{b}_{k}$ for $k=1,2,3$.

The position of $O$ and the orientation of $B$ in $A$ depend on the particular choice of the floating îrame. For the purposes of this paper, it is convenient to use the so-called Bückens frame [3]. This frame is defined by the constraint equations

$$
\sum_{i=1}^{N} m^{i}\left(\mathbf{r}^{i}+\mathbf{u}^{i}\right)=0
$$

and

$$
\sum_{i=1}^{N} m^{i}\left(\mathbf{r}^{i} \times \mathbf{u}^{i}\right)=0
$$

The constraints given by Eqs. (1) and (2) are easily accommodated by the finite element method. Moreover, the non rigid body, free-free modes of a structure automatically satisfy these conditions. This fact allows us to describe flexible motions in terms of the free-free mode shapes calculated from a finite element analysis.
The angular momentum, $\mathbf{H}$, of the system about its center of mass, $O$, is defined as

$$
\mathbf{H}=\sum_{i=1}^{N} m^{i}\left(\mathbf{r}^{i}+\mathbf{u}^{i}\right) \times \mathbf{v}^{i}
$$

where $\mathbf{v}^{i}$ denotes the velocity of the $i$ 'th particle in $A$. The system kinetic energy, $T$, is defined as

$$
T=\frac{1}{2} \sum_{i=1}^{N} m^{i} \mathbf{v}^{i} \cdot \mathbf{v}^{i}
$$

Using a basic kinematical relationship, one obtains

$$
\mathbf{v}^{i}=\mathbf{v}^{O}+\dot{\mathbf{u}}^{i}+\boldsymbol{\omega} \times\left(\mathbf{r}^{i}+\mathbf{u}^{i}\right)
$$

where $v^{O}$ is the velocity of $O$ in $A$ and () denotes the time derivative in $B$.

The angular momentum principle states the the time derivative of $\mathbf{H}$ in $A$ is equal to the net moment, $\mathbf{M}$, acting on the system about $O$. Thus,

$$
\dot{\mathbf{H}}+\boldsymbol{\omega} \times \mathbf{H}=\mathbf{M}
$$

Substitution of Eqs. (3) and (5) into Eq. (6), accounting for the constraints given by Eqs. (1) and (2), and linearizing the result yields

$$
\left[\begin{array}{ccc}
I_{11} & I_{12} & I_{13} \\
I_{12} & I_{22} & I_{23} \\
I_{13} & I_{23} & I_{33}
\end{array}\right]\left(\begin{array}{c}
\dot{\omega}_{1} \\
\dot{\omega}_{2} \\
\dot{\omega}_{3}
\end{array}\right)=\left(\begin{array}{c}
M_{1} \\
M_{2} \\
M_{3}
\end{array}\right)
$$

where

$$
\begin{array}{ll}
I_{11}=\sum_{i=1}^{N} m^{i}\left[\left(r_{2}^{i}\right)^{2}+\left(r_{3}^{i}\right)^{2}\right] & I_{12}=-\sum_{i=1}^{N} m^{i} r_{1}^{i} r_{2}^{i} \\
I_{22}=\sum_{i=1}^{N} m^{i}\left[\left(r_{3}^{i}\right)^{2}+\left(r_{1}^{i}\right)^{2}\right] & I_{23}=-\sum_{i=1}^{N} m^{i} r_{2}^{i} r_{3}^{i} \\
I_{33}=\sum_{i=1}^{N} m^{i}\left[\left(r_{1}^{i}\right)^{2}+\left(r_{2}^{i}\right)^{2}\right] & I_{13}=-\sum_{i=1}^{N} m^{i} r_{3}^{i} r_{1}^{i}
\end{array}
$$

Notice that Eq. (7) is equivalent to the linear form of Euler's equations and is decoupled from translational and flexible motions.

An assumed displacement expansion of the form

$$
u_{k}^{i}=\sum_{j=1}^{n} q_{j} \phi_{k}^{j i}
$$

is used to account for spacecraft flexibility. Defining the vector $\phi^{j}$ as

$$
\phi^{j}=\left[\phi_{1}^{j 1} \phi_{2}^{j 1} \phi_{3}^{j 1} \ldots \phi_{1}^{j N} \phi_{2}^{j N} \phi_{3}^{j N}\right]^{T},
$$


the strain energy, $U$, of the spacecraft is expressed as the quadratic form

$$
U=\frac{1}{2} q^{T} \bar{K}
$$

where

$$
\begin{aligned}
& q=\left[q_{1}, \ldots, q_{n}\right]^{T} \\
& \bar{K}_{l m}=\phi^{i T} K \phi^{m}
\end{aligned}
$$

and $K$ is the stiffness matrix associated with a finite element model of the spacecraft.

The equations governing flexible motion are obtained from the Lagrangian equations

$$
\frac{d}{d t}\left(\frac{\partial T}{\partial \dot{q}_{j}}\right)-\frac{\partial T}{\partial q_{j}}+\frac{\partial D}{\partial \dot{q}_{j}}+\frac{\partial U}{\partial q_{j}}=Q_{j} \quad(j=1, \ldots, n)
$$

where $D$ is the Rayleigh dissipation function, assumed to be of the classical form

$$
D=\frac{1}{2} \dot{q}^{T} \bar{C} \dot{q}
$$

The generalized force $Q_{j}$ in Eq. (14) is given by

$$
Q_{j}=\phi^{j T} f
$$

where

$$
f=\left[f_{1}^{1} f_{2}^{1} f_{3}^{1} \ldots f_{1}^{N} f_{2}^{N} f_{3}^{N}\right]^{T}
$$

and $f_{k}^{i}$ denotes the force acting on the $i$ 'th particle in the direction $\mathbf{b}_{k}$.

Substitution of Eqs. (4), (5), (9) and (11) into Eq. (14), accounting for the constraints given by Eqs. (1) and (2), and linearizing the result yields

$$
\bar{M} \ddot{q}+\bar{C} \dot{q}+\bar{K} q=\left[Q_{1} \ldots Q_{n}\right]^{T}
$$

where

$$
\bar{M}_{l m}=\sum_{i=1}^{N} \sum_{k=1}^{3} m^{i} \phi_{k}^{l i} \phi_{k}^{m i}
$$

When non rigid body, free-free modes of the spacecraft are used for the $\phi$ 's in Eq. (9), the matrices $\bar{M}$ and $\bar{K}$ are diagonal. Under the additional assumption of modal damping, the matrix $\vec{C}$ is also diagonal. In this case, Eq. (18) assumes the simple form

$$
m_{j j} \ddot{q}_{j}+c_{j j} \dot{q}_{j}+k_{j j} q_{j}=Q_{j} \quad(j=1, \ldots, n)(20)
$$

In summary, the equations of motion governing the rigid body and flexible motions are given by Eqs. (7) and (20), respectively. All of the coefficients appearing in these equations can be obtained in a straightforward manner from a finite element analysis of the spacecraft. We also note that these equations continue to hold when concentrated moments and rotational inertias are included in the development.

The orientation of $B$ in $A$ is governed by the kinematical differential equations

$$
\begin{aligned}
& \dot{\epsilon}_{1}=\frac{1}{2}\left(\omega_{1} \epsilon_{4}-\omega_{2} \epsilon_{3}+\omega_{3} \epsilon_{2}\right) \\
& \dot{\epsilon}_{2}=\frac{1}{2}\left(\omega_{1} \epsilon_{3}+\omega_{2} \epsilon_{4}-\omega_{3} \epsilon_{1}\right) \\
& \dot{\epsilon}_{3}=\frac{1}{2}\left(-\omega_{1} \epsilon_{2}+\omega_{2} \epsilon_{1}+\omega_{3} \epsilon_{4}\right) \\
& \dot{\epsilon}_{4}=-\frac{1}{2}\left(\omega_{1} \epsilon_{1}+\omega_{2} \epsilon_{2}+\omega_{3} \epsilon_{3}\right)
\end{aligned}
$$

where $\epsilon_{1}, \ldots, \epsilon_{4}$ are Euler parameters [4].

\section{Examples}

A sketch of the spacecraft model used in the example problems is shown in Figure 2. The spacecraft bus is modeled as a rigid body of mass $m_{b}$ with principal mass moments of inertia $I_{1}, I_{2}$, and $I_{3}$. Unit vectors $c_{1}, c_{2}$, and $c_{3}$ are fixed in the bus and are aligned with the principal axes. Two massless beam elements of length $L$ with tip masses of $m_{p}$ are used to inodel flexible solar panels. The axes of the undeformed beams are assumed to be aligned with the $\mathbf{c}_{1}$ direction and pass through the bus mass center. The first principal plane of bending for the beams is defined by a vector normal $\mathbf{n}$. The normal to the second principal plane of bending is then defined as the cross product $\mathbf{c}_{1} \times \mathbf{n}$. Flexural rigidities for bending in the two principal planes are denoted by $(E I)_{1}$ and $(E I)_{2}$. Moments $M_{1}, M_{2}$, and $M_{3}$ are applied to the spacecraft bus in the $\mathbf{c}_{1}, \mathbf{c}_{2}$, and $c_{3}$ directions, respectively. Each moment is subject to the inequality constraints $-C \leq M_{k} \leq C$ for $k=1,2,3$.

The example problems deal with rest-to-rest maneuvers whereby the spacecraft is slewed from an initial orientation defined by $\epsilon_{1}=\epsilon_{2}=\epsilon_{3}=0$, $\epsilon_{4}=1$ to a final orientation defined by $\epsilon_{k}=\epsilon_{k f}$ for $k=1, \ldots, 4$. The goal is to reorient the spacecraft and bring it to a quiescent state in a specified time $T$ while minimizing the performance index

$$
\Gamma=\int_{0}^{T}\left(M_{1}^{2}+M_{2}^{2}+M_{3}^{2}\right) d t
$$




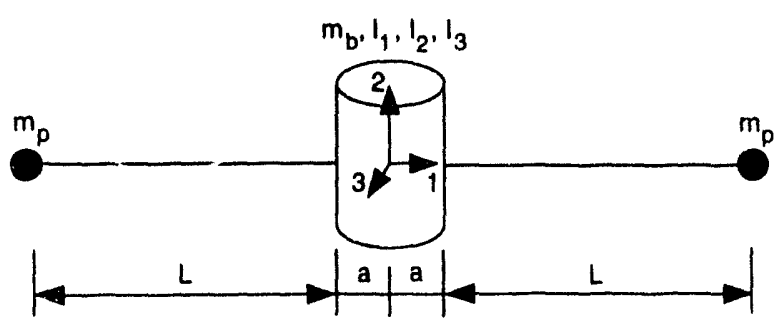

Figure 2: Sketch of model used in examples.

Since closed form solutions to Eqs. (21-24) do not exist in general, it is necessary to make use of numerical procedures to solve the optimal control problem. In this paper we utilize a dynamic programming algorithm recently applied to trajectory planning problems in robotics [5]. The salient features of the algorithm are:

1. Quadratic convergence nearby the solution.

2. Inequality constraints for inputs accounted for exactly.

3. Equality constraints for states imposed using a quadratic penalty function.

4. Optimality conditions determined from definiteness of $p$ by $p$ matrices.

5 . Order $N n p$ storage required for each iteration.

6. Order $N n^{3}$ operations required for each iteration.

In the above description, $n$ is the number of states, $p$ the number of inputs, and $N$ the number of time steps used to discretize the problem.

Values for the dimensionless parameters used in the examples are provided in Table 1. Two antisymmetric bending modes are retained in the assumed displacement expansion (see Eq. (9)). For this simple model, these are the only two modes excited by the applied moments. The free-free mode shapes for the examples are mass matrix normalized and were calculated using the commercial finite element code MSC/NASTRAN. Results presented in Figures 3-5 are plotted as functions of the dimensionless time variable $\tau=t / T$. The generalized coordinates $q_{1}$ and $q_{2}$ are associated with deformation nominally in the first and second principal planes of bending, respectively.

The first example is concerned with a maneuver where reorientation of the spacecraft can be accomplished simply by slewing about the 2 -axis of

\begin{tabular}{|c|c|}
\hline$a / L$ & 0.50 \\
$m_{p} / m_{b}$ & 0.06 \\
$I_{2} / I_{1}$ & 0.50 \\
$I_{3} / I_{1}$ & 1.00 \\
$m_{p}(a+L)^{2} / I_{1}$ & 1.38 \\
$(E I)_{2} /(E I)_{1}$ & 3.00 \\
$(E I)_{1} T^{2} /\left(m_{p} L^{3}\right)$ & 150 \\
$C T^{2} /\left[I_{2}+2 m_{p}(a+L)^{2}\right]$ & 8.00 \\
\hline
\end{tabular}

Table 1: Parameters used in examples.
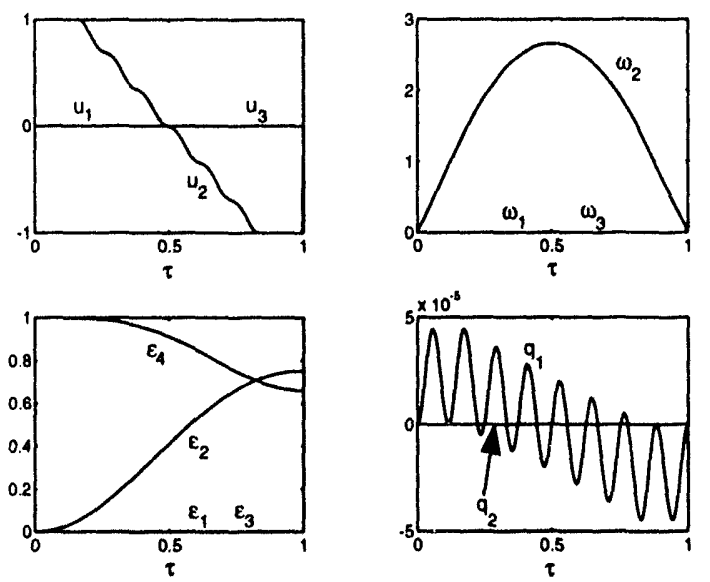

Figure 3: Results for first example.

the bus. In this example, the normal $\mathbf{n}$ is chosen as $c_{3}$ so that the principal planes of bending are aligned with the principal axes of the spacecraft. The final orientation of the satellite is defined by the Euler parameters $\epsilon_{1 f}=\epsilon_{3 f}=0, \epsilon_{2 f}=\sin (\theta / 2)$, and $\epsilon_{4 f}=\cos (\theta / 2)$ where $\theta=1.7 \mathrm{rad}$. This reorientation corresponds to a rotation of 1.7 radians about the 2-axis of the bus. Plots of the dimensionless moments $u_{k}=M_{k} / C(k=1,2,3)$, angular velocity measure numbers, Euler parameters, and generalized coordinates are shown in Figure 3. As expected, only $u_{2}$ is nonzero and out-of-plane vibrations are absent as indicated by the constant zero value of $q_{2}$. Notice also the antisymmetry of $u_{2}$ about $\tau=1 / 2$.

The second example is identical to the first with the exception that the normal $\mathbf{n}=\left(\mathbf{c}_{2}+\mathbf{c}_{3}\right) / \sqrt{2}$. This choice for $\mathbf{n}$ corresponds to a 45 degree misalignment of the principal planes of bending with the principal axes of the spacecraft. Plots of the results are shown in Figure 4. Notice that both out-of-plane moments and vibrations occur. We note that it is still possible to achieve the rest-to- 

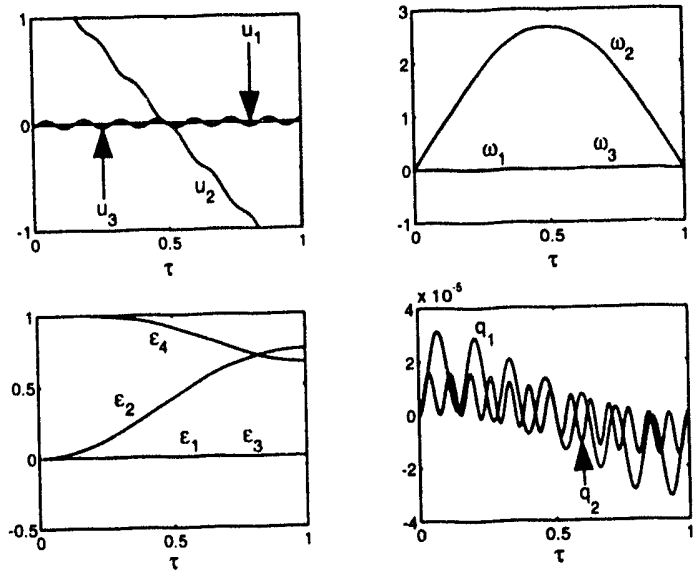

Figure 4: Results for second example.

rest maneuver simply with a moment about the 2-axis of the bus, but this is not an optimal maneuver. It may also occur that as the angle of rotation $\theta$ is increased the maneuver is only possible with nonzero values for $M_{1}$ and $M_{3}$. Otherwise, the inequality constraints on $M_{2}$ may be prohibitively stringent. Although it is difficult to see the behavior of the other two inputs from the figure, $u_{1}$ is symmetric and $u_{3}$ is antisymmetric about $\tau=1 / 2$.

The third evample is identical to the second with the exception that the final orientation is defined by the Euler parameters $\epsilon_{1 f}=0$, $\epsilon_{2 f}=\epsilon_{3 f}=\sin (\theta / 2) / \sqrt{2}$, and $\epsilon_{4 f}=\cos (\theta / 2)$ where $\theta=1.7 \mathrm{rad}$. This example examines a situation in which the maneuver could be accomplished by slewing about a principal axis of bending not aligned with a principal axis of the spacecraft. If the optimal maneuver was such, then $\omega_{1}$ would be zero for all times. This, however, is clearly not the case as is evident from the results presented in Figure 5 ; Notice also the symmetries of the inputs about $\tau=1 / 2$.

\section{Summary}

A method is presented for input shaping for 3$D$ slew maneuvers of flexible spacecraft. The method is applicable to a wide range of spacecraft and does not require the derivation of equations of motion for special purpose models. Once a
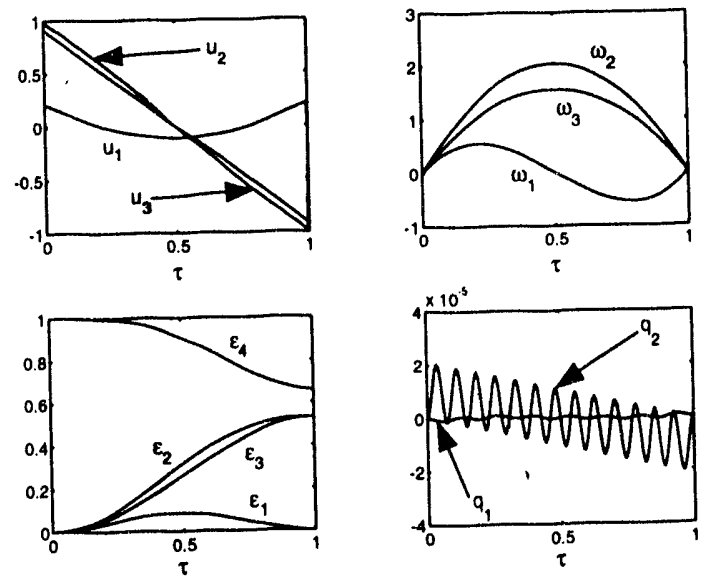

Figure 5: Results for third example.

finite element eigenanalysis is performed, the formulation and solution for input shaping can be accomplished in a straightforward manner. The effect of misalignment of the principal mass axes with the principal planes for bending in a simple spacecraft model is investigated. Symmetries in the inputs observed previously for planar maneuvers are also shown to be present in the general 3. D case.

\section{References}

[1] T. Singh and S.R. Vadali, "Input-Shaped Control of Three- Dimensional Maneuvers of Flexible Spacecraft," Journal of Guidance, Control, and Dynamics, Vol. 16, No. 6, 1993, pp. 1061-1068.

[2] J. Ben-Asher, J.A. Burns, and E.M. Cliff, "Time-Optimal Slewing of Flexible Spacecraft," Journal of Guidance, Control, and Dynamics, Vol. 15, No. 2, 1992, pp. 360-367.

[3] Canavin, J.R., and Likins, P.W., "Floating Reference Frames for Flexible Spacecraft," Journal of Spacecraft and Rockets, Vol. 14, No. 12, 1977, pp. 724-732.

[4] T.R. Kane, P.W. Likins, and D.A. Levinson, Spacecraft Dynamics, McGraw-Hill, New York, 1983.

[5] C.R. Dohrmann and R.D. Robinett, "Robot Trajectory Planning via Dynamic Programming," to be presented at the Fifth International Symposium on Robotics and Manufacturing, Maui, Hawaii, August 1994. 
11

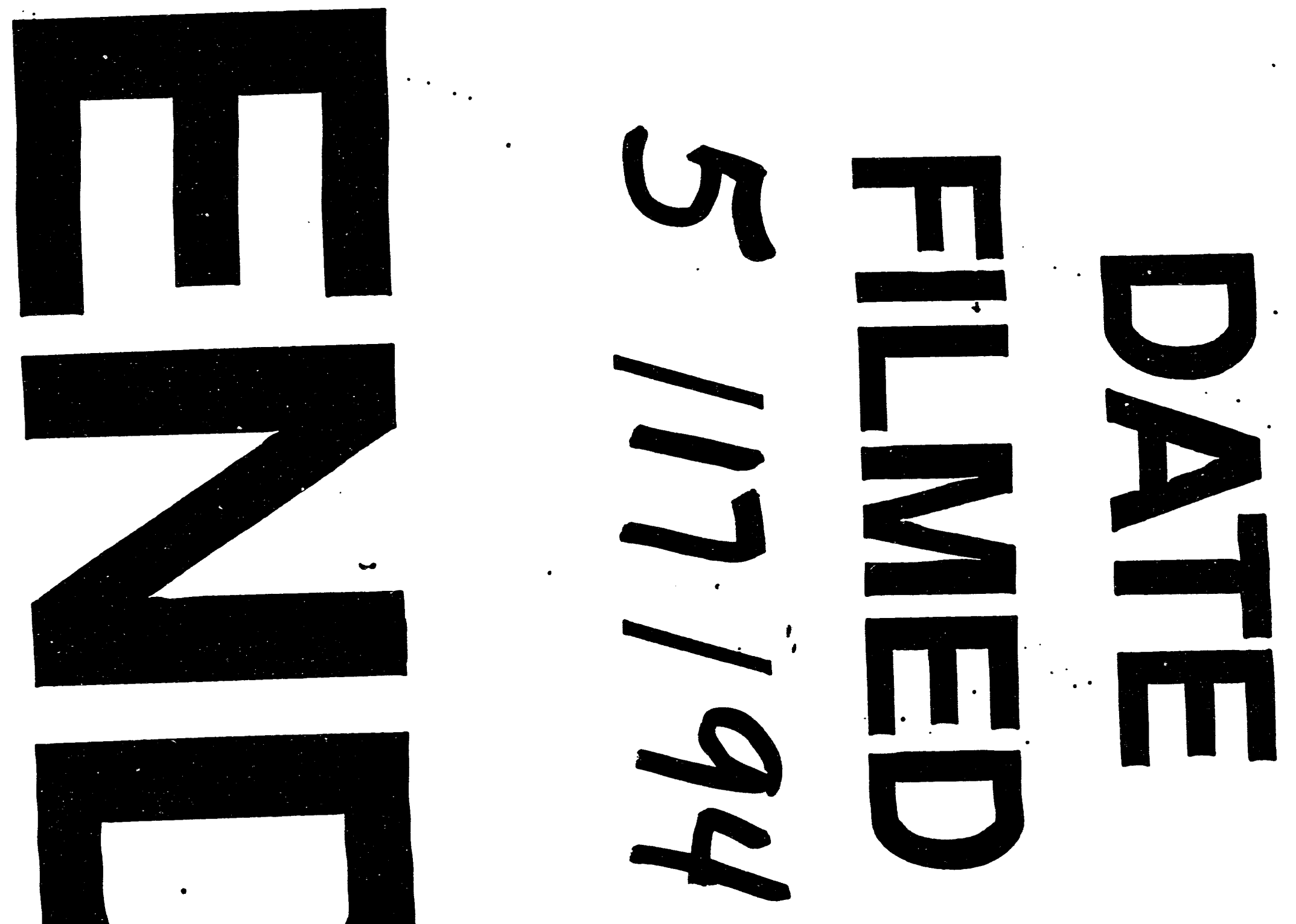


$+1$ 